\title{
Factors Contributing to Drug Resistant Tuberculosis: A Case Study of Tuberculosis Patients Attending Rift Valley Provincial General Hospital Nakuru, Kenya
}

\author{
Lucia Kipkoech Keter ${ }^{1,}$, , Silas Kandie Cherogony ${ }^{2}$, Richard Kipserem Korir ${ }^{1}$, Charles Mutai ${ }^{1}$ \\ ${ }^{1}$ Kenya Medical Research Institute, Mbagathi Road, NAIROBI, Kenya \\ ${ }^{2}$ Kenya Medical Training College, NAIROBI, Kenya
}

Email address:

lketer@yahoo.co.uk (L. K. Keter), sk.cherogony@gmail.com (S. K. Cherogony), richkori@gmail.com (R. K. Korir), charlesmutai@hotmail.com (C. K. Mutai)

\section{To cite this article:}

Lucia Kipkoech Keter, Silas Kandie Cherogony, Richard Kipserem Korir, Charles Mutai. Factors Contributing to Drug Resistant Tuberculosis: A Case Study of Tuberculosis Patients Attending Rift Valley Provincial General Hospital Nakuru, Kenya. Science Research.

Vol. 3, No. 3, 2015, pp. 66-73. doi: 10.11648/j.sr.20150303.14

\begin{abstract}
Tuberculosis remains a major cause of morbidity and mortality in Kenya. The main challenges compounding Tuberculosis control include drug resistant tuberculosis, Human Immunodeficiency Virus /Acquired Immunodeficiency Syndrome (HIV/AIDS) co-infections and the declining funding for tuberculosis control among others. Factors such as non-adherence to therapy, poor drug quality or poor prescribing practices have been reported to contribute to Drug Resistance TB. The study aimed at establishing factors that contribute to development of Drug Resistant Tuberculosis among patients on TB treatment attending the Rift Valley Provincial General Hospital, Nakuru. The study employed a cross-sectional descriptive survey of patients with tuberculosis. Primary data was collected using semi structured questionnaire while secondary data was retrieved from the patient's treatment records. Clearance to conduct the study was sought from the Hospital Authorities and written consent obtained from the participants. Data was analyzed using computer Statistical Package for Social Science. Fifty three percent of the study participants were in the $21-40$ years age brackets while the male to female ratio was 5:4. Majority (61.3\%) of the patients were co-infected with HIV/AIDS and $42.5 \%$ were new smear positive pulmonary tuberculosis (PTB), $23.7 \%$ were smear negative PTB or Extra-Pulmonary tuberculosis (EPTB), $28.5 \%$ were retreatment and/ or relapse while $4.3 \%$ were chronic and/ or multi-drug resistant tuberculosis (MDR-TB). Eighty five percent of the participants adhered to treatment while patients beginning to feel better was cited as the major factor $(42.5 \%)$ leading to non-adherence to treatment. High percentage of tuberculosis - HIV/AIDS co-infection and high prevalence of tuberculosis among women was observed in this study. There need for impact assessment of the 2007/ 2008 post-elections violence on the spread of TB and development of MDR-TB in the affected parts of Rift Valley Province.
\end{abstract}

Keywords: Mycobacterium Tuberculosis, Drug Resistant, Treatment, Adherences, Rift Valley Provincial General Hospital

\section{Introduction}

Tuberculosis (TB) remains a major global health problem causing ill-health among millions of people each year and ranks as the second leading cause of death from infectious disease worldwide, after the human immunodeficiency virus (HIV). The World Health Organization (WHO) latest estimate indicates that 8.6 million people developed TB in 2012 and 1.3 million died from the disease. Among these deaths were an estimated 170, 000 from multi-drug resistant tuberculosis (MDR-TB), a relatively high total compared with 450000 incident cases of MDR-TB [1]. Most of the estimated number of cases in 2012 occurred in Asia (58\%) and the African Region (27\%) with South Africa been home to approximately $17-26 \%(0.4-0.6$ million) of the total African region cases. The current estimated epidemiological burden of TB in Kenya stands at 320 per 100,000 populations making it one of the 22 high TB burden countries in the world [1]. Tuberculosis usually affects the lungs (Pulmonary TB), but it can also attack any organ of the body and when TB occurs outside the lungs it is referred to as Extra-Pulmonary TB (EPTB). It is transmitted from person to person via droplets from the throat and lungs of a person with a positive sputum smear and over $90 \%$ of people infected 
with the tubercle bacillus will not develop TB disease [2]. Those at risk of developing the disease following infection with the tubercle bacillus include children under one year, poorly nourished individuals, and those with poor immune defenses such as persons infected with HIV, diabetics, alcoholics, patients with leukemia and patients receiving immunosuppressive therapies [3].

Tuberculosis treatment involves the use of multiple drugs taken in combination which are available as fixed dose combinations (FDC). The Kenya's National TB treatment guidelines 2005 recommended a Category I treatment regimen encompassing 2 months daily supervised intensive phase with Rifampicin (R)+Isoniazid (H)+Pyrazinamide (Z)+Ethambutol (E) followed by 4 months continuation phase of daily self-administered of Rifampicin and Isoniazid or 6 months of Ethambutol (E) and Isoniazid (H) (2RHZE/4RH or $2 \mathrm{RHZE} / 6 \mathrm{EH}$ ) for new smear-positive and new severely ill cases and Category II regimen for retreatment cases entailing 2 months of SRHZE (Streptomycin, S) followed by one month of RHZE and then five months of RHE (2SRHZE/1RHZE/5RHE). Category III regimen which entail 2 months of RHZE and 4 months of RH (2RHZE/4RH) is recommended for new smear negative PTB and EPTB [3]. In order to promote total adherence to treatment, Direct Observation of Treatment (DOT) is encouraged through a treatment supporter who could be a health care worker, a family member or a community volunteer. The WHO recommended DOT strategy was adopted in Kenya over 2 decades ago and DOT as a strategy entails that medication is taken in while the care provider is observing the patient swallowing the drug. However, diagnosing TB patients at a health facility and sending them to continue taking their treatment at home poses serious challenges for other members of the households who assist the patients with treatment and this compromise on adherence. Adherence to a medication regimen is generally defined as the extent to which patients take medications as prescribed by their health care providers. Poor adherence to medication regimens accounts for substantial worsening of disease, death, and increased health care costs [4].

The resurgence of new strains of mycobacterium such as multi-drug resistant TB (MDR TB) which are resistant to two first line TB drugs (Isoniazid and rifampicin) seriously threatens the control and prevention efforts and is associated with both high death rates and treatment costs $[1,5,6]$. Resistance is caused by a genetic mutation that makes a drug ineffective against the mutant bacilli. Factors such as poor adherences to therapy, poor drugs quality or poor prescribing practice like insufficient dosage and inadequate duration of therapy have been reported to contribute to development of resistance to therapies [7]. The recommended treatment for MDR-TB consisting of 6 months intensive phase with Pyrazinamide, Ethionamide, Capreomycin, Cycloserine, Ofloxacin and Ethambutol under strict DOT (DOTS-Plus) and preferably in hospital followed by 18 - 24 month continuation phase with Ofloxacin, Cycloserine, Ethionamide and Pyrazinamide depending on culture conversion to negative [7-9]. Multi-drug resistant TB treatment is more complex, involving use of expensive drugs and prolonged therapy period with more adverse events [6].

Poor adherence to treatment remains a major obstacle in the global fight against TB and the reasons for non-adherence are complex and polygonal involving more than the patients' personal characteristics and attitudes. Studies have reported low literacy levels, household members' fear of catching the disease, discriminatory behavior by health care providers, delays in care seeking behavior and self-denial due to stigma experienced by TB patients as some of the challenges facing adherence to TB treatment $[10,11]$. Additionally, TB like HIV/AIDS is often associated with stigmatization leading to non-compliance to the treatment regime, which may create resistance to treatment $[11,12]$. Therefore, the type of care received at both the health facility and the homes has a strong bearing on the reactions patients may have and their adherence to treatment. The Kenyan government through the Ministry of Health has supported a sustained public education campaign through the media on the symptoms of TB, mode of transmission, the importance of seeking treatment and the fact that TB is curable. To further step up the fight, the government has also put a lot of effort in ensuring adequate availability of drugs and adequately trained staff in all government and selected mission hospitals. However, its current effort to find, treat and cure everyone who gets ill with the disease is not sufficient. The prevalence of MDR-TB seems to be rising with 7,976 reported re-treatment cases of which 249 tested positive for both isoniazid and rifampicin resistance [13] and in order to arrest the rising cases of drug resistant $\mathrm{TB}$, it is important to determine the possible contributing factors to the high prevalence MDR-TB cases. Untreated or improperly treated patients with DR-TB are a source of ongoing transmission of resistant strains, resulting in future added costs and mortality. Therefore the study aimed at establishing the factors contributing to development of DR-TB among patients on TB treatment attending Rift Valley Provincial General Hospital, Nakuru.

\section{Materials and Methods}

\subsection{Study Population}

A cross sectional survey was carried out among TB patients attending Rift Valley Provincial General Hospital (PGH), Nakuru town in Nakuru District (currently Nakuru County), Rift Valley Province in 2008. Nakuru District covers a total area of 7,291 Sq-Km and a population of approximately 1.4 million people of whom 5,618 are infected with TB [14]. According to the Kenya's National Leprosy and Tuberculosis Program (NLTP), Nakuru is one of the five Kenya's largest urban Centres with high TB notification cases made it suitable for the study [3].The patients attending Rift Valley PGH are referred from dispensaries and Health Centres within the town Council and surrounding Districts such as Koibatek to the North, Baringo to the North East, Laikipia to the East, Narok 
to the West and Olkalou to the South (Figure 1). The patients diagnosed with TB are treated and referred back to their respective districts for continuation of treatment. However, some of these patients are referred back to the TB clinic at the Provincial Hospital when the condition worsens.

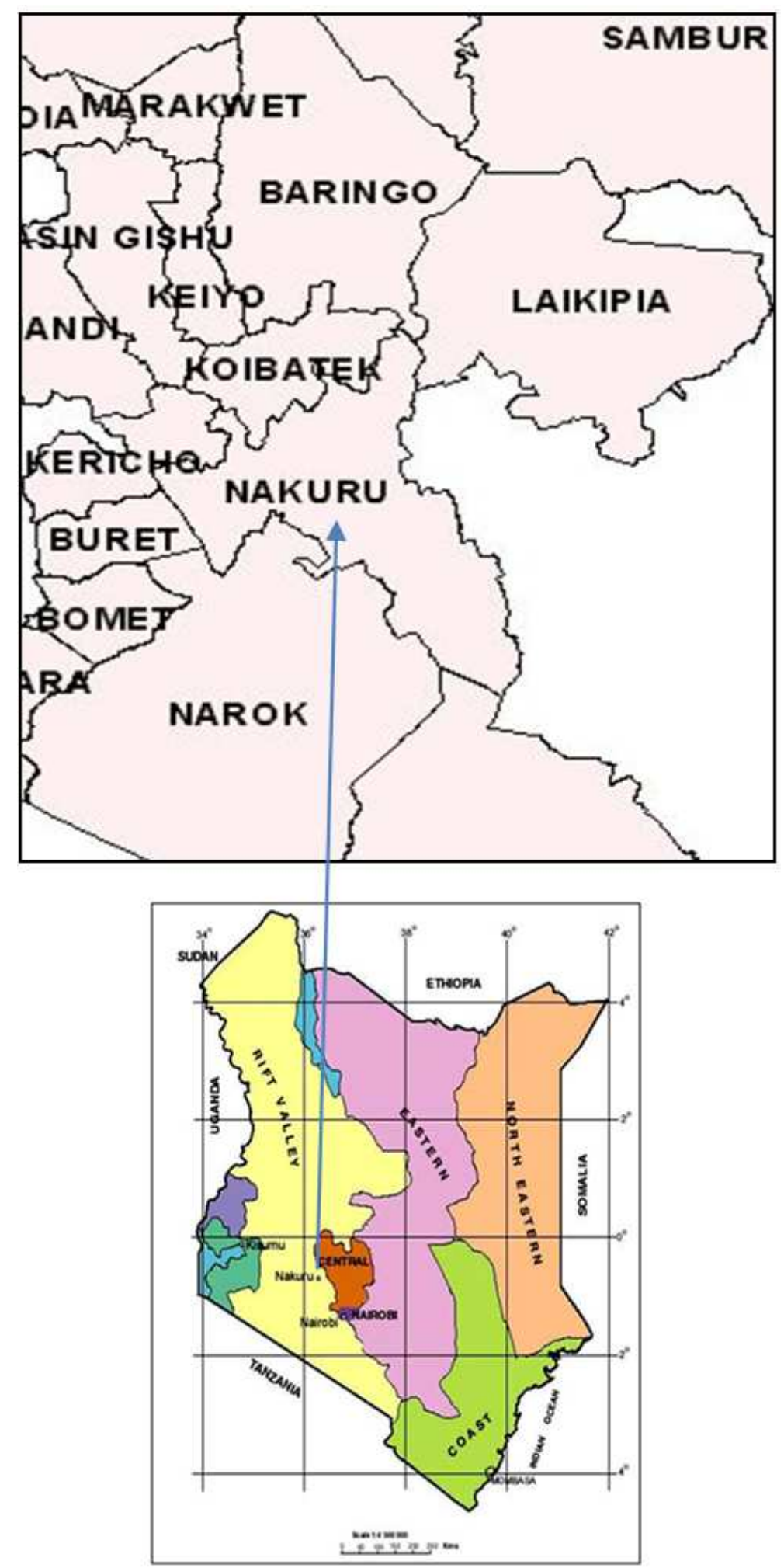

Figure 1. A map of Nakuru and the surrounding Districts.

\subsection{Study Sample}

Non-probability purposive sampling method due to its familiarity and convenience of collecting data was used to select participants. All patients diagnosed with TB, on anti-tubercular drugs and must have been on treatment for at 
least 1 month, and attending TB clinic at Rift Valley PGH were selected. Patients co-infected with HIV/AIDS and other medical risk conditions were also included in the study. A sample size of 218 participants calculated using Fishers formulae and putting into consideration the proportion of bacteriological confirmed cases of smear positive and re-treatments, smear negative and extra-pulmonary TB [3] were identified.

\subsection{Sampling Procedure}

Patients attending the consulting TB clinic at the Rift Valley PGH were informed of the study objectives and recruited into the study upon leaving the Doctors' consultation rooms. Those willing to participate signed an informed consent form and were then interviewed using a short pre-tested questionnaire. The interviews educed information on the socio-demographic information of the participants for instance age, sex, marital status, education level and occupation, and other information like participants treatment adherence, reasons for non-adherence to treatment, factors associated with treatment failure, duration of treatment and knowledge on TB transmission, signs and symptoms among others. The patients' medical files were also scrutinized and appropriate data such as participants' case investigation and definition on entry, treatment phase and regimen and co-infections was retrieved and recorded.

\subsection{Data Analysis}

The data captured in the questionnaires was grouped and examined for errors, then cleaned, entered into a summary sheet and analyzed using Computer Statistical Package for Social Science (SPSS) Version 20. Association of variables was done using correlation and regression analysis to establish relationship between independent and dependent variables. All patients presenting sputum smear positive $(\mathrm{sm}+)$ after one month of intensive phase of first line regimen were considered to have Drug Resistant Tuberculosis, while those presenting with sputum smear positive $(\mathrm{sm}+)$ after two months of intensive phase of first line regimen and have $85-100 \%$ adherence level were considered to have MDR-TB. Smear positive patient at 5 and 8 month of treatment were also considered to have MDR-TB.

\subsection{Ethical Considerations}

The authority to conduct this research was sought from the study participants, the Provincial Medical Officer of Health, the Rift Valley PGH Medical Superintendent and the TB clinics in-charge. Before the questionnaires were administered, the objectives of the study and the planned use of the information were explained to the study participants and were assured of confidentiality and anonymity. In addition, participants were assured that no person-identifiers would be used for both data collection and publication. Permission was then sought and written consent obtained in all cases.

\section{Results}

\subsection{Socio- Demographic Characteristics of the Participants}

The socio-demographic characteristics of the TB patient population that participated in the survey are presented in Table 1. The oldest participant was 76 years while the youngest was 5 years. Majority $(52.7 \%)$ of the study participants were in the age bracket of $21-40$ years with $32 \%$ falling in the 31-40 year age bracket. More than half (56.5\%) of the participants were males while $43.5 \%$ were females resulting in a male to female ratio of around 5:4. Fifty two percent of the study participants were single while $47.8 \%$ were married. All the participants with the exception of 2 had attained at least primary school level of education however, $57 \%$ of the participants reported to have no formal employment.

Table 1. Socio-demographic characteristics of the study Participants ( $n=$ 186)

\begin{tabular}{|c|c|c|}
\hline \multirow{2}{*}{ Demographic characteristics } & \multicolumn{2}{|c|}{ Total number of participants } \\
\hline & Frequency & Percent \\
\hline \multicolumn{3}{|l|}{ Sex } \\
\hline Male & 105 & 56.5 \\
\hline Female & 81 & 43.5 \\
\hline \multicolumn{3}{|l|}{ Age (Years) } \\
\hline$\leq 10$ & 20 & 10.8 \\
\hline $11-20$ & 25 & 13.4 \\
\hline $21-30$ & 39 & 21.0 \\
\hline $31-40$ & 59 & 31.7 \\
\hline $41-50$ & 29 & 15.6 \\
\hline $51-60$ & 9 & 4.8 \\
\hline$\geq 61$ & 5 & 2.7 \\
\hline \multicolumn{3}{|l|}{ Marital status } \\
\hline Married & 89 & 47.8 \\
\hline Single & 97 & 52.2 \\
\hline \multicolumn{3}{|l|}{ Education Level } \\
\hline Nil/ None & 2 & 1.1 \\
\hline Primary & 56 & 30.1 \\
\hline Secondary & 110 & 59.1 \\
\hline Tertiary colleges & 18 & 9.7 \\
\hline \multicolumn{3}{|l|}{ Primary Occupation } \\
\hline Peasant farmer / Business & 24 & 12.9 \\
\hline Formal employment & 56 & 30.1 \\
\hline Un-employed & 106 & 57.0 \\
\hline
\end{tabular}

\subsection{Participants' Investigation and Case Definition}

Ninety nine percent of the study participants had undergone both Sputum analysis and Radiological chest $\mathrm{x}$-ray (CXR) to confirm the diagnosis while blood analysis was applied in only $1 \%$ of the cases. The participants' investigation at entry revealed that $29.6 \%$ presented with smear negative or EPTB while $70.4 \%$ were PTB (Table 2). The reported clinical classification at entry revealed that $42.5 \%$ were new smear positive PTB while $28.5 \%$ were re-treatment cases of which $10.8 \%$ were smear positive PTB relapses, $4.8 \%$ were treatment failure, $5.9 \%$ re- treatment after default and the remaining were smear negative PTB or EPTB cases previously 
treated. Sixty-one percent of the participants were co-infected with HIV/AIDS while $36.5 \%$ were not co-infected.

Table 2. Case definition at treatment entry, treatment regimen and adherence of the study participants $(n=186)$

\begin{tabular}{|c|c|c|}
\hline \multirow{2}{*}{ Characteristics } & \multicolumn{2}{|c|}{ Total number of participants } \\
\hline & Frequency & Percent \\
\hline \multicolumn{3}{|l|}{ Reported form of TB } \\
\hline Pulmonary Tuberculosis (PTB) & 131 & 70.4 \\
\hline Smear negative or EPTB & 55 & 29.6 \\
\hline \multicolumn{3}{|l|}{ Reported clinical classification of TB } \\
\hline New smear positive PTB & 79 & 42.5 \\
\hline New smear negative or EPTB & 44 & 23.7 \\
\hline Re-treatment/ Relapse & 53 & 28.5 \\
\hline smear positive PTB relapses & 20 & 10.8 \\
\hline Treatment failure & 9 & 4.8 \\
\hline Re-treatment after default & 11 & 5.9 \\
\hline Smear negative $P T B$ or EPTB & & \\
\hline $\begin{array}{l}\text { cases previously treated } \\
\text { Other TB cases }\end{array}$ & 13 & 7.0 \\
\hline Chronic/MDR TB & 8 & 4.3 \\
\hline Transfers & 2 & 1.1 \\
\hline \multicolumn{3}{|l|}{ Co-Infections with Tuberculosis } \\
\hline HIV/ AIDS & 114 & 61.3 \\
\hline Diabetes & 2 & 1.1 \\
\hline Cancer (Leukemia) & 2 & 1.1 \\
\hline None & 68 & 36.5 \\
\hline \multicolumn{3}{|l|}{ Treatment regimen } \\
\hline Category I & 122 & 65.6 \\
\hline Category II & 17 & 9.1 \\
\hline Category III & 44 & 23.7 \\
\hline Category IV & 8 & 4.3 \\
\hline \multicolumn{3}{|l|}{ Treatment adherence level } \\
\hline $\begin{array}{l}\text { Missed between } 1 \text { and } 3 \text { weeks in a } \\
\text { month }\end{array}$ & 3 & 1.6 \\
\hline $\begin{array}{l}\text { Missed between } 4 \text { and } 7 \text { days in a } \\
\text { month }\end{array}$ & 10 & 5.4 \\
\hline $\begin{array}{l}\text { Missed between } 2 \text { and } 3 \text { days in a } \\
\text { month }\end{array}$ & 15 & 8.1 \\
\hline Missed once in a month & 32 & 17.2 \\
\hline Never missed & 126 & 67.7 \\
\hline
\end{tabular}

\subsection{Treatment Adherence and knowledge on TB Transmission}

Some of the TB signs and symptoms according to the participants included cough (34.9\%), fever (33.4\%), weight loss $(25.3 \%)$, night sweat $(30.1 \%)$ and difficulty in breathing while enlarged lymph nodes was the least reported $(2.7 \%)$. The participants' knowledge on mode of transmission included from mother to child, sleeping in the same room with TB patient, sharing utensils, infected persons coughing directly at others. To determine treatment adherence level, participants were asked on the number of missed treatment within the past one month of treatment. Grading was based on the number of days of missed treatment. Between 1 and 3 weeks was considered poor, more than 4 days but less than 7 days was considered fair, between 2 and 3 days was considered fairly good, once a month good and those who have never missed were graded as very good. Majority $(67.7 \%)$ of the participants reported that they had never missed their medications since treatment initiation while 3 participants reported to have missed their treat for between 1 and 3 weeks (Table2).

Twenty percent of the participants were in their initial month of the therapy and $17.2 \%$ were in their final month. The rest were within 2 to 7 months of treatment (Figure 2). The most frequently perceived reasons for non-adherence according to the participants (Table 3) were forgetfulness (50\%), adverse effects $(21.4 \%)$, once one start feeling better (42.5\%), substance abuse (14.5\%) and fear of discrimination at $12.4 \%$.

Table 3. Perceived reasons for non-adherence to the TB therapy

\begin{tabular}{lcc}
\hline Factors & Frequency & Percentage \\
\hline TB drugs too strong & 49 & 21.4 \\
Once they start feeling better & 79 & 42.5 \\
Fear of discrimination & 23 & 12.4 \\
Cultural beliefs & 15 & 8.1 \\
Running out of drugs & 13 & 7.0 \\
Lack of drugs at the clinic & 0 & 0 \\
Denial of suffering from TB & 3 & 1.6 \\
Substance abuse & 27 & 14.5 \\
Social mobility & 8 & 4.3 \\
Forgetting to take & 93 & 50.0 \\
Religious beliefs & 8 & 4.3 \\
\hline
\end{tabular}

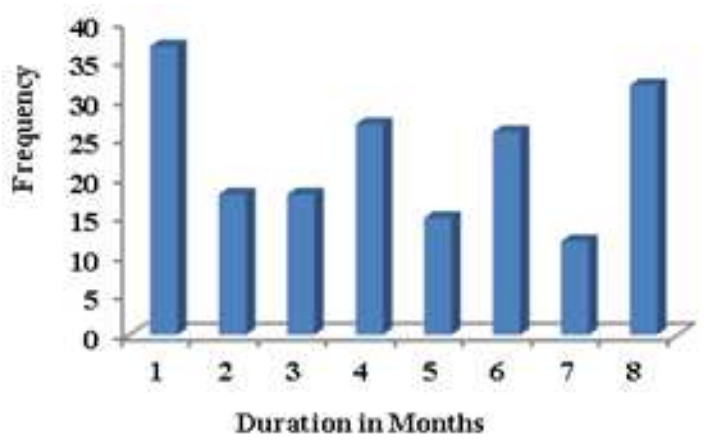

Figure 2. Participants duration on treatment $(n=186)$

\subsection{Treatment Adherence and Knowledge on TB Transmission}

The most mentioned factors contributing to unsuccessful TB treatment according to the study participants were HIV/ AIDS (95.7\%), displacement (85.5\%) and substance abuse such as alcoholism and smoking (79.6 \%) as shown in Figure 3.

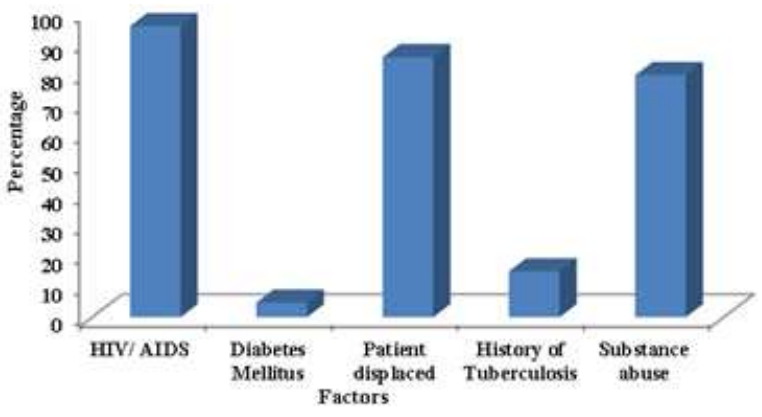

Figure 3. Factors associated with TB treatment failure according to the participants (Figures cannot add up because the question allowed for multiple responses) 


\section{Discussion}

Kenya has a large and rising TB disease burden and is ranked among the twenty-two countries that collectively contribute about $80 \%$ of the world's TB cases [8]. The large increase in TB in Kenya is attributed primarily to the Human Immunodeficiency Virus (HIV) and the high poverty levels with consequent socio-economic deprivation. This is most evident in urban areas such as Nakuru where there has been a phenomenal growth of slums hence increase in slum population. The large urban slum population has been followed closely by an increase in the proportion of $\mathrm{TB}$ notification cases [3].

The Socio-Demographic Characteristics of the study participants indicated that $52.7 \%$ were in the $21-40$ years age bracket with $32.0 \%$ of these falling in the range of $31-40$ years of age which is the young economically productive age groups. Other studies have reported a rapid rise in TB morbidity and mortality among this young adult population mostly between 15 - 44 years of age $[1,3]$ which is in line with the study findings. Slightly more than half $(56.5 \%)$ of the participants interviewed were males while $43.5 \%$ were females. These shows a significantly higher percentage of females affected by TB in the study population with a male to female ratio of 1.3 which varies with the current WHO male to female global ratio of 1.7 but more closer to the Kenya's MOH report of 2005 showing that males are 1.4 time more likely to have TB than females $[1,3]$. The high frequency of female observed in the study could be attributed to the way of life of the community within the study site who were displaced during the post-election violence that occurred in late 2007 and early 2008, where majority of women and children remained in congested camps unlike men who move out of camps. The study also revealed that $52 \%$ of the participants were married while the remaining were not married. Significantly high percentage literacy level was recorded among the study participants. Only 2 out of 186 had no formal education, $30.1 \%$ had attained primary school education while $128(68.8 \%)$ of the participants had at least secondary school level of education. High literacy level has been reported to positively impact on slowing rapid development of DR-TB because a great degree of literacy has been positively associated with good health perception [15].

Studies have shown that poverty compels people to live in overcrowded environment with poorly ventilated rooms, conditions known to favour increased TB prevalence. The study revealed that $57 \%$ of the participants were unemployed, $12.9 \%$ engaged in peasant farming or some form of self-employment while $30.1 \%$ were in formal employment. Majority of these participants had no source of income. The Kenya Demographic and Health Survey of 2003 ranked the district as among the poorest in the country [16]. There was a significant positive correlation between socio-economic status/ poverty and MDR-TB $(\mathrm{r}=+0.592, \mathrm{p}<0.01)$ that was observed. This suggested that poor participant experiences higher prevalence of MDR-TB which is attributed to low disposable income as a result of low income thus increasing the likelihood for such households' failure to adopt appropriate and effective TB treatment. Studies have shown that poor socio-economic status leads to overcrowding, slum development, poor nutrition and limited access to health services which are factors identified to contributing to the increasing TB burden. Poor socio-economic conditions also contribute to non-adherence to $\mathrm{TB}$ treatment hence the emergence of MDR-TB [17].

Ninety nine percent of the respondents were investigated for both sputum smear analysis and chest X-ray to confirm the diagnosis because HIV/AIDS patient are known to have smear negative or extra-pulmonary Tuberculosis. Table 2 shows the distribution of the different types of TB among the study participants. Thirty percent of the participants presented with smear negative or EPTB whereas $70 \%$ were PTB. In regards to the reported clinical classification of the TB, $42.5 \%$ of the total cases were new smear positive, $23.7 \%$ were new smear negative or EPTB and the remaining $33.8 \%$ were re-treatment and others such as treatment transfers whose history was unknown and chronic and or MDR TB. Sputum smear positive cases are the most infectious and thus of the highest priority from a public health perspective. The WHO report of 2010 listed Kenya among the countries with low percentage of notified cases of sputum smears positive PTB. The $42.5 \%$ smear positive recorded in this study was in line with the 2009 case notification that reported $46 \%$ [8]. Re-treatment cases felt into the smear positive PTB relapse $(10.8 \%)$, smear negative PTB and EPTB (7.0\%), treatment failure (4.8\%), re-treatment after default (5.9\%) and others which included 2 cases of treatment after transfer and $8(4.3 \%)$ participants categorized as either chronic or multi drug resistant TB. Nearly a third of the total reported forms of TB were smear negative PTB or EPTB and a quarter of the re-treatment cases were recurrent smear negative PTB or EPTB cases. With high prevalence of HIV among the study participants it is possible that some of the cases were not true TB cases but represented undiagnosed HIV-related diseases. Twenty percent of the participants were in their initial month and $17.2 \%$ were in their final month of therapy while the remaining $62.9 \%$ were spread between the 2nd and 7th month of therapy (Figure 2). Despite the good adherence level recorded in this study, the re-treatment cases as a result of defaulting were $5.9 \%$ which was higher than the $1.6 \%$ cases categorized as poor adherence.

Only $66.4 \%$ (87 out of 131) smear positive participants were screened for sputum analysis after 2 months of intensive phase of TB therapy and the laboratory results showed that only $66.7 \%$ (58 out of 87 ) had been converted to smear negative an indication of some resistance to the Isoniazid-rifampicin regimen. The percentage of resistance could have been higher considering 33.6\% (44 out of 131) of the smear positive participants were not investigated for sputum analysis. The Sputum analysis at 5 and 8 months of treatment was not included in the study due to study time limitation. Other parameters such as weight on entry revealed that a large proportion of the participants $(51.6 \%)$ weighed between $51-60 \mathrm{~kg}$ on entry giving a mean weight of $62.2 \mathrm{~kg}$. 
After 2 months of treatment $52.9 \%$ of the participants weighed between 51-60 kg a slight improvement from the entry weight as shown by the increase in mean weight from $62.2 \mathrm{~kg}$ to $68 \mathrm{~kg}$. The government should take stringent measures to ensure TB patients on treatment are screened according to the guidelines to ensure early detection of treatment failure for timely intervention.

The high burden of TB in Kenya has mainly been attributed to the high prevalence of HIV, estimated at $7.1 \%$ from Sentinel surveillance of 2010 [1]. This study revealed that $61.3 \%$ of the participants were co-infected with HIV/AIDS while 2 persons each were suffering from diabetes and leukemia whereas a third of the participants were not co-infected. Majority of those co-infected with both HIV and TB were youths of age brackets $25-40$ years. The prevalence of HIV/AIDS worldwide has been reported to be high among the youth within the above age bracket while the HIV/AIDS prevalence in Kenya is estimated at $7.1 \%$ of the general population [8]. According to the WHO Global tuberculosis Report of 2010, $41 \%$ of TB patients in Kenya had HIV co-infection, which is $20 \%$ below the findings of this study. Tuberculosis is the most frequent opportunistic infection detected among HIV patients and the interaction between the TB and the HIV epidemics is particularly fatal because TB adds to the illness burden of HIV-infected persons and shortens their life expectancy, while the HIV epidemic stimulates TB spread $[5,18]$. The high mortality rate of HIV patients having TB further leads to the community's loss of confidence in the curability of TB [18] and increases the level of non-adherence to treatment. However, Tuberculosis treatment success rate in Kenya, according to the 2009 study, stood at $85 \%$ for new smear positive pulmonary TB cases despite the high prevalence of HIV among TB patients in the country [8]. HIV/AIDS infection also predisposes TB patients to development of drug resistant TB [19-21] and the study outcome indicated a strong correlation between MDR-TB and HIV/AIDS co-infection and other medical risk factors resulting in a positive significant relationship $(\mathrm{r}=$ $+0.254, \mathrm{p}<0.01$ ). This suggested that high prevalence of HIV/AIDS co-infection was not only predisposing patients to $\mathrm{TB}$, but also raises the chances of developing MDR-TB.

The participants reported that sleeping in the same room with a TB patient, sharing utensils and TB infected person coughing directly at others are responsible for the spread of TB infections. Although strict separating of eating and drinking utensils for $\mathrm{TB}$ patients contributes to general hygienic practice, it can hardly prevent TB transmission, but results from this study confirmed this popular belief. Many participants knew that TB patients should cover their mouth whenever they cough. Some of the common symptoms which accompany the infection such as coughing for more than three weeks, fever, weight loss and night sweat were reported by the participants. However, enlarged lymph node was least known. Various studies have reported that adequate knowledge among TB patients is important in order to check the spread of the diseases within the community and also improve on adherence to treatment $[10,17]$.
Poor adherence to treatment remains a major obstacle in the global fight against TB and the reasons for non-adherence are multifaceted [1]. According to this study, $84.9 \%$ of the participants adhered to treatment compared to only $1.6 \%$ who had poor adherence level to treatment while $13.5 \%$ showed adherence levels ranging from fairly to good (Table 2 ). The results showed that the study population had high adherence levels that may been attributed to participants' adequate knowledge of TB treatment and the good health education administered by the health care personnel on importance of adherence to treatment. It could also be attributed to the patient treatment support in the community which currently stands at $88 \%$ countrywide [1]. Non-adherence to TB treatment has been reported to undermine the effective control of TB and the ability of health care providers to recognize non-adherence has been reported to be inadequate [4]. Factors such as once one started feeling better $(42.5 \%)$, treatment side effects $(21.4 \%)$, substance abuse (Cigarette smokers, alcohol consumption and others) $14.5 \%$, fear of discrimination and stigmatization $(12.4 \%)$, cultural beliefs $(8.1 \%)$, religious beliefs (4.3) and social mobility (4.3\%) among others were cited by the participants to negatively contribute to failure to taking their TB drugs as required under DOT strategy. Also, TB is strongly associated with HIV/AIDS by community members because of the perceived mode of transmission and also many symptoms of TB, such as weight loss, fever, and chronic cough, are associated with AIDS labeling most HIV-negative TB patients as AIDS cases, which lead to patients' stigmatization and discrimination. Most of these factors can be eliminated when the community is well equipped with adequate knowledge. To improve on adherence among TB patients in Kenya, the Government has launched several new approaches such as community based DOTS (CB-DOTS), Public -Private Mix for DOTS, collaboration between TB and HIV control programs, Prevention with Positives (PwP) strategy and the development of an elaborate advocacy, communication and social mobilization strategy aimed at influencing communities to seek early care when TB symptoms occur and to remain on treatment until this is completed when treatment is initiated [3]. The TB treatment adherence level shows negative significant association with MDR - TB $(\mathrm{r}=-.679 *, \mathrm{P}<0.05)$. This implies that the observed high treatment adherence levels among the respondents significantly reduce the prevalence of MDR - TB among TB patients in the study area. In regards to possible causes of TB treatment failure, $95.7 \%$ of the participants blamed it on HIV/AIDS, substance abuse $(79.6 \%)$, patient's displacement $(85.5 \%)$, previous history of TB (15.1\%) and Diabetes $(4.8 \%)$.

\section{Conclusion}

The provision of diagnosis and treatment according to the DOTS / Stop TB Strategy has resulted in major achievements in TB care and control [1]. However, TB continued to pose a major public health challenge with huge new TB cases reported globally and most of these TB cases occur among 
men though the burden of disease among women is also high has observed in this study. There was also a high $(61.3 \%)$ TB - HIV co-infections among the study participants. More work needs to be carried out to determine the impact of the 2007/ 2008 post-elections violence on the spread of TB and development of MDR-TB in Nakuru and other affected parts of Rift Valley Province.

\section{Acknowledgements}

The authors wish to thank the study participants, the Provincial Medical Officer of Health, the Medical Superintendent, the TB clinics in-charge and all the staff of Rift Valley PGH for their valuable contributions.

\section{References}

[1] World Health Organization Global Tuberculosis Report 2013. www.who.int (Accessed on 5th May 2014).

[2] Warker R. and Edward C. The Chemotherapy of Tuberculosis Clinical Pharmacy and Therapeutic. 3rd Edition. Churchill Livingstone. 2004, pp 583-93.

[3] Ministry of Health: National Leprosy and Tuberculosis Guideline (MOH/NLTP), 2005 http://www.nltp.co.ke/docs/National_NLTP_Guideline.pdf (Accessed on March 19th, 2014)

[4] Osterberg L. and Blaschke T. Adherence to Medication: A Review. New England Journal of Medicine 2005, 353: 487 497.

[5] Zignol M., Hosseini M.S., Wright A., Lambregts-van Weezenbeek C., Nunn P., Watt C.J., Williams B.G., and Dye C. Global incidence of Multi-Drug resistant Tuberculosis. Journal of infectious diseases. 2006, 194(4):479 - 485 .

[6] Chan E.D., Laurel V., Strand M.J., Chan J.F., Mai-Lan N. H., Goble M., and Iseman M. D. Treatment and Outcome Analysis of 205 Patients with Multidrug-resistant Tuberculosis. American Journal of Respiratory and Critical Care Medicine 2004, 169(10): 1103 - 1109 .

[7] World Health Organization, 2008. Guidelines for the programmatic management of drug-resistant tuberculosis. Emergency update 2008. WHO/HTM/TB/2008.402 http://whqlibdoc.who.int/publications/2008/9789241547581_e ng.pdf

[8] World Health Organization Report, 2010. Global Tuberculosis Control. www.who_reports/reports/Global_Tuberculosis_Control_2010 .pdf (Accessed on 5th May 2014).
[9] Ministry of Public Health and Sanitation (MPHS), 2010. Guidelines for the Management of Drug Resistant Tuberculosis in Kenya. Division of Leprosy, Tuberculosis and Lung Disease, Ministry of Public Health and Sanitation.

[10] Slama K., Tachfouti N., Obtel M., Nejjari C. Factors associated with treatment default by tuberculosis patients in Fez, Morocco. Eastern Mediterranean Health Journal 2013, 19(8): 687 - 693.

[11] Kaona F.A.D., Tuba M., Siziya S. and Sikaona L. An assessment of factors contributing to treatment adherence and knowledge of TB transmission among patients on TB treatment. BMC Public Health 2004, 4:68

[12] Odusanya O.O. and Babafeni O.J. Patterns of delays among pulmonary tuberculosis patients in Lagos, Nigeria. BMC Public Health 2004, 4: 17.

[13] Ministry of Health: National Leprosy and Tuberculosis Guideline (MOH/NLTP), 2006. National Leprosy and Tuberculosis Program Guidelines. Nairobi, Kenya

[14] Ministry of Health: National Leprosy and Tuberculosis Guideline (MOH/NLTP) Annual Report, 2007. Nairobi, Kenya

[15] Ponyk R.M., Makhubele M.B., Hargreaves J.R., Tollman S.M., Hausler H.P. Assessing health seeking behaviour among tuberculosis patients in South Africa. International Journal of Tuberculosis and Lung Disease 2001, 5(7): 619 - 627.

[16] Kenya Demographic and Health Survey (KDHS), 2003. Kenya Central Bureau of Statistics (CBS), Ministry of Health (MOH) Kenya, and ORC Macro. 2004. Calverton, Maryland: CBS, $\mathrm{MOH}$, and ORC Macro.

[17] Saira Z., Tyaba H., Khawaja T.M. Socioeconomic Factors Contributing to Multidrug-Resistant Tuberculosis (MDR-TB). Journal of Biomedical Science and Research 2010, 2 (4): 279-283.

[18] Ngamvithayapong J., Winkvist A. and Diwan V. High AIDS awareness may cause tuberculosis patient delay: results from an HIV epidemic area, Thailand. AIDS 2000, 14: 1413 -1419.

[19] Frieden T.R. and Sbarbaro J.A. Promoting adherence to treatment for tuberculosis: the importance of direct observation. Bulletin of the World Health Organization 2007, 85(5): 407-409.

[20] Sharma S.K., Mohan A. Multidrug-Resistant Tuberculosis: A Menace That Threatens To Destabilize Tuberculosis Control. Chest 2006, 130(1): $261-272$.

[21] Patrice J., Patrice S., Severine F., Kye Seng G., Christophe S., David W.H., Warren D.J., Nalin R., Pape J.W., Fitzgeral D.W. Multidrug-resistant tuberculosis at an HIV testing center in Haiti. AIDS 2006, 20: 415 - 418. 